\title{
Pseudo Laguerre Matrix Polynomials, Operational Identities and Quasi-Monomiality
}

\author{
Maged G. Bin-Saad ${ }^{*}$, M. A. Pathan ${ }^{2}$ \\ ${ }^{1}$ Department of Mathematics, Aden University, Kohrmakssar, Yemen \\ ${ }^{2}$ Centre for Mathematical and Statistical Sciences (CMSS), KFRI, Thrissur, India \\ Email: *mgbinsaad@Yahoo.com, mapathan@gmail.com
}

How to cite this paper: Bin-Saad, M.G. and Pathan, M.A. (2018) Pseudo Laguerre Matrix Polynomials, Operational Identities and Quasi-Monomiality. Advances in $\mathrm{Li}$ near Algebra \& Matrix Theory, 8, 87-95. https://doi.org/10.4236/alamt.2018.82008

Received: October 30, 2017

Accepted: June 8, 2018

Published: June 11, 2018

Copyright ( 92018 by authors and Scientific Research Publishing Inc. This work is licensed under the Creative Commons Attribution International License (CC BY 4.0).

http://creativecommons.org/licenses/by/4.0/

\begin{abstract}
The main purpose of this paper is to introduce the matrix extension of the pseudo Laguerre matrix polynomials and to explore the formal properties of the operational rules and the principle of quasi-monomiality to derive a number of properties for pseudo Laguerre matrix polynomials.
\end{abstract}

\section{Keywords}

Pseudo Laguerre Matrix Polynomials, Lowering Operators, Raising Operators, Quasi-Monomiality, Operational Rules

\section{Preliminaries and Definitions}

In the last two decade, matrix polynomials have become more important and some results in the theory of classical orthogonal polynomials have been extended to orthogonal matrix polynomials see for instance [1]-[7]. Orthogonal matrix polynomials are important from both the theoretical and practical points of view, they appear in connection with representation theory, matrix expansion problems, prediction theory and in the matrix quadrature integration problems, see for example [5] [8] [9]. Numerous problems of chemistry, physics and mechanics are related to second order matrix differential equation. Moreover, some properties of the Hermite and Laguerre matrix polynomials and a generalized form of the Hermite matrix polynomials have been introduced and studied in [4] [9]-[19]. Other classical orthogonal polynomials as Gegenbauer, Chebyshev, Jacobi and Konhauser polynomials have been extended to orthogonal matrix polynomials, and some results have been investigated, see for example [9] [18] [19] [20] [21]. We say that a matrix $A$ in $\mathbb{C}^{N \times N}$ is a positive stable if $\mathfrak{R}(\lambda)>0$ for all $\lambda \in \sigma(A)$, where $\sigma(A)$ is the set of the eigenvalues of $A$. If 
$A_{0}, A_{1}, \cdots, A_{n}, \cdots$ are elements of $\mathbb{C}^{N \times N}$ and $A_{n} \neq 0$, then we call

$$
P(x)=A_{n} x^{n}+A_{n-1} x^{n-1}+A_{n-2} x^{n-2}+\cdots+A_{1} x+A_{0},
$$

a matrix polynomial of degree $n$ in $x$. If $A+n I$ is invertible for every integer $n \geq 0$ then

$$
(A)_{n}=A(A+I)(A+2 I) \cdots(A+(n-1) I) ; n \geq 0,(A)_{0}=I .
$$

Thus we have

$$
(A)_{n}=\Gamma^{-1}(A) \Gamma(A+n I) .
$$

For any matrix $A$ in $\mathbb{C}^{N \times N}$, we have the following relation [22]

$$
(1-x)^{-A}=\sum_{n=0}^{\infty} \frac{(A)_{n} x^{n}}{n !},|x|<1 .
$$

Next, we recall that the Konhauser matrix polynomials are defined in [21] as

$$
Z_{n}^{(A, \lambda)}(x ; k)=\frac{\Gamma(A+(k n+1) I)}{n !} \sum_{s=0}^{n}(-1)^{s}\left(\begin{array}{l}
n \\
s
\end{array}\right) \Gamma^{-1}(A+(k s+1) I)(\lambda x)^{k s} .
$$

In [23] Dattoli et al. introduced the two variable pseudo Laguerre polynomials $L_{n}(x, y ; k, j)$ in the form:

$$
L_{n}(x, y ; k, j)=n ! \sum_{s=0}^{n} \frac{(-1)^{s} y^{n-s} x^{k s+j}}{s !(n-s) !(k s+j) !} .
$$

In this work, we construct a matrix version of the pseudo Laguerre matrix polynomials given by (1.4) as follows:

Definition 1.1. Let $A$ be a matrix in $\mathbb{C}^{N \times N}$ satisfying the condition $\mathfrak{R}(\lambda)>-1$ for every $\lambda \in \sigma(A), k \in \mathbb{Z}^{+}$and $|t|<1$. We define the pseudo-Laguerre matrix polynomials by the series

$$
L_{n}(x, y ; k, A)=n ! \sum_{s=0}^{n} \frac{(-1)^{s} y^{n-s} x^{A+k s I}}{s !(n-s) !} \Gamma^{-1}(A+(k s+1) I) .
$$

The relevant generating function for the polynomials $L_{n}(x, y ; k, A)$ can be obtained by the method suggested in [23], thus getting

Theorem 1.1. Let $A$ be a matrix in $\mathbb{C}^{N \times N}$ satisfying the condition $\mathfrak{R}(\lambda)>-1$ for every $\lambda \in \sigma(A), k \in \mathbb{Z}^{+}$, and $|t|<1$. Then

$$
\sum_{n=0}^{\infty} L_{n}(x, y ; k, A) \frac{t^{n}}{n !}=x^{A} \mathrm{e}^{y t} C_{A}\left(x^{k} t ; k\right),
$$

where

$$
C_{A}(x ; k)=\sum_{s=0}^{\infty} \frac{(-1)^{s} x^{s}}{s !} \Gamma^{-1}(A+(k s+1) I) .
$$

being the matrix version of the Tricomi function defined in (see [4]).

Proof. If we use the series (1.7) in right-hand side of (1.6), we get

$$
x^{A} \mathrm{e}^{y t} C_{A}\left(x^{k} t ; k\right)=\sum_{n, s=0}^{\infty} \frac{(-1)^{s} x^{k s+A} y^{n}}{s !} \Gamma^{-1}(A+(k s+1) I) \frac{t^{n+s}}{n !} .
$$


Now, by letting $n \rightarrow n-s$, we obtain the left-hand side of the assertion (1.6).

We must emphasize that the matrix polynomials in (1.6) are a generalized form of Konhauser matrix polynomials defined by (1.3) and indeed we have

$$
Z_{n}^{(A, \lambda)}(y ; k)=\frac{(\lambda y)^{-A} \Gamma(A+(k n+1) I)}{n !} L_{n}(\lambda y, 1 ; k, A) .
$$

For the purpose of this work we introduce the following matrix version of Kampé de Fériet double hypergeometric series $F_{l: m ; n}^{p: q ; k}[x, y]$ and matrix version of the generalized hypergeometric function ${ }_{p} F_{q}$ [24] as follows:

$$
\begin{aligned}
& F_{l: m ; n}^{p: q ; k}\left[\begin{array}{l}
\left(A_{p}\right):\left(B_{q}\right) ;\left(C_{k}\right) ; x, y \\
\left(D_{l}\right):\left(E_{m}\right) ;\left(F_{n}\right) ;
\end{array}\right]=\sum_{r, s=0}^{\infty} \frac{\prod_{j=1}^{p}\left(A_{j}\right)_{r+s} \prod_{j=1}^{q}\left(B_{j}\right)_{r} \prod_{j=1}^{k}\left(C_{j}\right)_{s}}{r ! s !} \\
& {\left[\prod_{j=1}^{l}\left(D_{j}\right)_{r+s}\right]^{-1}\left[\prod_{j=1}^{m}\left(E_{j}\right)_{r}\right]^{-1}\left[\prod_{j=1}^{n}\left(F_{j}\right)_{s}\right]^{-1} x^{r} y^{s}}
\end{aligned}
$$

and

$$
{ }_{p} F_{q}\left[A_{1}, \cdots, A_{p} ; B_{1}, \cdots, B_{q} ; x\right]=\sum_{n=0}^{\infty} \frac{\left(A_{1}\right)_{n} \cdots\left(A_{p}\right)_{n}\left(B_{1}\right)_{n} \cdots\left(B_{q}\right)_{n}}{n !} x^{n} .
$$

In view of the definition (1.9) and the definition of the matrix version of the Gauss multiplication theorem

$$
(A)_{k n}=k^{k n}\left(\frac{A+j I-I}{k}\right)_{n},
$$

it is not difficult to show that

$$
L_{n}(x, y ; k, A)=x^{A} y^{n} \times_{1} F_{k}[-n ; \Delta(k ; A+I) ; x],
$$

where throughout this work $\Delta(m ; A+I)$ denotes the array of $m$ parameters $\frac{A}{m}, \frac{A+I}{m}, \cdots, \frac{A+(m-1) I}{m}, m>0$. For an arbitrary matrix $A \in \mathbb{C}^{N \times N}$ the following two formulas are well-known consequences of the derivative operator $\hat{D}_{x}$ and the integral $\hat{D}_{x}^{-1}[18]$

$$
\begin{aligned}
& \hat{D}_{x}^{n} x^{A}=\Gamma(A+I) \Gamma^{-1}(A-(n+1) I) x^{A-n I}, \\
& \hat{D}_{x}^{-n} x^{A}=\Gamma(A+I) \Gamma^{-1}(A+(n+1) I) x^{A+n I},
\end{aligned}
$$

where $\hat{D}_{x}=\frac{\partial}{\partial x}$ and $n \in \mathbb{N} \bigcup\{0\}$.

Note that, in this work we apply the concept of the right-Riemann-Liouville fractional calculus to obtain operational identities and relations. Motivated by the works mentioned above, we aim in this work to present systematic investigation of the matrix version of the pseudo Laguerre polynomials given by (1.5) and exploit methods of operational nature and the monomiality principle to derive a number of operational representations, operators and generating functions con- 
structed matrix polynomials in (1.5).

\section{Operational Identities and Quasi-Monomiality}

First of all, we establish the following operational representations for pseudo Laguerre matrix polynomials $L_{n}(x, y ; k, A)$.

Theorem 1.1. Let $A$ be a matrix in $\mathbb{C}^{N \times N}$ satisfying the condition $\mathfrak{R}(\lambda)>-1$ for every $\lambda \in \sigma(A)$ and $k \in \mathbb{Z}^{+}$. Then

$$
L_{n}(x, y ; k, A)=\Gamma^{-1}(A+I) \mathrm{e}^{-\hat{D}_{x}^{-k} \hat{D}_{y}}\left\{x^{A} y^{n}\right\} .
$$

Proof. In view of (1.10) and (1.11), we have

$$
\frac{\partial^{s}}{\partial y^{s}} y^{n}=\frac{n !}{(n-s) !} y^{n-s},
$$

and

$$
\Gamma^{-1}(A+I) \hat{D}_{x}^{-k s} x^{A}=\Gamma^{-1}(A+(k s+1) I) x^{A+k s},
$$

The desired result now follows by applying the identities (2.2) and (2.3) to the definition (1.5).

Theorem 2.2. Let $A$ be a matrix in $\mathbb{C}^{N \times N}$ satisfying the condition $\mathfrak{R}(\lambda)>-1$ for every $\lambda \in \sigma(A)$ and $k \in \mathbb{Z}^{+}$. Then

$$
L_{n}(x, y ; k, A)=\Gamma^{-1}(A+I)\left(y-\hat{D}_{x}^{-k}\right)^{n}\left\{x^{A}\right\} .
$$

Proof. The result follows directly from the formula

$$
(x-y)^{n}=\sum_{s=0}^{\infty} \frac{(-n)_{s}}{s !} x^{n-s} y^{s},
$$

the assertion (2.3) and the definition (1.5)

The use of the monomiality principle has offered a powerful tool for studying the properties of families of special functions and polynomials. We know that according to the monomiality principle [23] [25], a polynomial set $\left\{p_{n}(x)\right\}, n \in \mathbb{N}$ is quasi-monomial, if there exist two operators $\hat{M}$ and $\hat{P}$, called multiplicative and derivative operators respectively, which when acting on the polynomials $\left\{p_{n}(x)\right\}$ yield [25]

$$
\begin{aligned}
& \hat{M}\left\{p_{n}(x)\right\}=p_{n+1}(x), \\
& \hat{P}\left\{p_{n}(x)\right\}=n p_{n-1}(x) .
\end{aligned}
$$

The operators $\hat{M}$ and $\hat{P}$ satisfy the commutation relation:

$$
[\hat{M}, \hat{P}]=\hat{1}
$$

and thus display a Weyl group structure. If $\hat{M}$ and $\hat{P}$ have differential realization, then the differential equations satisfied by $p_{n}(x)$ are

$$
\begin{aligned}
& \hat{M} \hat{P}\left\{p_{n}(x)\right\}=n p_{n}(x), \\
& \hat{P} \hat{M}\left\{p_{n}(x)\right\}=(n+1) p_{n}(x) .
\end{aligned}
$$

In this regard, the matrix polynomial set $L_{n}(x, y ; k, A)$ is quasi-monomial 
under the action of the multiplicative operator

$$
\hat{M}=y+\frac{x^{A+I}}{k} \frac{\partial}{\partial x} x^{-A} D_{y}^{-1},
$$

and the derivatives operators

$$
\begin{gathered}
\hat{P}_{1}=\frac{-1}{k} \frac{\partial^{k}}{\partial x^{k}} x^{A+I} \frac{\partial}{\partial x} x^{-A}, \\
\hat{P}_{2}=\frac{\partial}{\partial y} .
\end{gathered}
$$

According to the quasi-monomiality properties, we have

$$
\begin{aligned}
& \hat{M}\left\{L_{n}(x, y ; k, A)\right\}=L_{n+1}(x, y ; k, A) \\
& \hat{P}_{1}\left\{L_{n}(x, y ; k, A)\right\}=n L_{n-1}(x, y ; k, A) \\
& \hat{P}_{2}\left\{L_{n}(x, y ; k, A)\right\}=n L_{n-1}(x, y ; k, A) .
\end{aligned}
$$

Therefore, the identities

$$
\begin{aligned}
& \hat{M} \hat{P}_{1}\left\{L_{n}(x, y ; k, A)\right\}=(n+1) L_{n}(x, y ; k, A) \\
& \hat{M} \hat{P}_{2}\left\{L_{n}(x, y ; k, A)\right\}=(n+1) L_{n}(x, y ; k, A)
\end{aligned}
$$

in differential forms give us

$$
\begin{gathered}
{\left[\hat{D}_{x}^{k} x^{A+I} \frac{\partial}{\partial x} x^{-A}\left(I-A+y \frac{\partial}{\partial y} I\right)+x \hat{D}_{x}^{k+1} x^{A+I} \frac{\partial}{\partial x} x^{-A}-k n \frac{\partial}{\partial y} I\right] L_{n}(x, y ; k, A)=0} \\
{\left[y \frac{\partial}{\partial y} I+\frac{x^{A+I}}{k} \frac{\partial}{\partial x} x^{-A} I-n I\right] L_{n}(x, y ; k, A)=0 .}
\end{gathered}
$$

Moreover, regarding the Lie bracket [,] defined by $[A, B]=A B-B A$, we led to

$$
\begin{aligned}
& {\left[\hat{P}_{1}, \hat{M}\right] L_{n}(x, y ; k, A)=L_{n}(x, y ; k, A),} \\
& {\left[\hat{P}_{2}, \hat{M}\right] L_{n}(x, y ; k, A)=L_{n}(x, y ; k, A) .}
\end{aligned}
$$

From the lowering operators $\hat{P}_{1}$ and $\hat{P}_{2}$ in (2.6) and (2.7), we can define operators playing the role of the inverse operators $\hat{P}_{1}^{-1}$ and $\hat{P}_{2}^{-1}$ (see [[8], Equation (15)]). Thus, we get

$$
\begin{gathered}
\hat{P}_{1}^{-1}=-k x^{A} \hat{D}_{x}^{-1} x^{-(A+I)} \hat{D}_{x}^{-k}, \\
\hat{P}_{2}^{-1}=\hat{D}_{y}^{-1},
\end{gathered}
$$

and they satisfy

$$
\hat{P}_{1}^{-1}\left\{L_{n}(x, y ; k, A)\right\}=\hat{P}_{2}^{-1}\left\{L_{n}(x, y ; k, A)\right\}=\frac{L_{n+1}(x, y ; k, A)}{(n+1)} .
$$

Clearly, we have

$$
\hat{P}_{1} \hat{P}_{1}^{-1}\left\{L_{n}(x, y ; k, A)\right\}=\hat{P}_{2} \hat{P}_{2}^{-1}\left\{L_{n}(x, y ; k, A)\right\}=L_{n}(x, y ; k, A) .
$$

Further, from (2.9)-(2.11), we can infer that $L_{n}(x, y ; k, A)$ are the natural 
solution of the following equation

$$
\left[\hat{D}_{y}^{-1}+k x^{A} \hat{D}_{x}^{-1} x^{-(A+I)} \hat{D}_{x}^{-k}\right] L_{n}(x, y ; k, A)=0 .
$$

Moreover, from (2.5) in conjunction with (2.8), we get

$$
L_{n+1}(x, y ; k, A)=y L_{n}(x, y ; k, A)+\frac{x^{A+I}}{k} \frac{\partial}{\partial x} x^{-A} \hat{D}_{y}^{-1} L_{n}(x, y ; k, A),
$$

which yields the following recurrence relation

$$
L_{n+1}(x, y ; k, A)-y L_{n}(x, y ; k, A)-L_{n}(x, y ; k, A+k I)=0 .
$$

Finally, let

$$
\hat{N}=y-\hat{D}_{x}^{-k},
$$

then upon using (2.4) one obtains by routine calculations

$$
\begin{aligned}
& \Gamma^{-1}(A+I)[y-\hat{N}]^{n}\left\{x^{A}\right\} \\
& =\sum_{s=0}^{\infty}(-1)^{s}\left(\begin{array}{l}
n \\
s
\end{array}\right) L_{n}(x, y ; k, A) y^{n-s}=\Gamma(A+k n I+I) x^{A+k n I} .
\end{aligned}
$$

\section{Generating Functions and Expansions}

First, in the identity (2.1) multiply throughout by $\frac{t^{n}}{n !}$, sum and then employ the formulae (1.10) and (1.11) and the result

$$
\mathrm{e}^{x}=\sum_{n=0}^{\infty} \frac{x^{n}}{n !}
$$

to get

$$
\begin{aligned}
& \mathrm{e}^{y t} x^{A}{ }_{o} F_{k}\left[-; \frac{A+I}{k}, \frac{A+2 I}{k}, \cdots, \frac{A+(k-1) I}{k} ;-(k x)^{k}\right] \\
& =\Gamma(A+I) \sum_{n=0}^{\infty} L_{n}(x, y ; k, A) \frac{t^{n}}{n !} .
\end{aligned}
$$

Next, let us consider the generating relation

$$
f^{(A, B)}(x, y, z, w ; k, l \mid t)=\sum_{n=0}^{\infty} L_{n}(x, y ; k, A) \times L_{n}(z, w ; l, B) \frac{t^{n}}{n !},
$$

which according to operational identity (2.4)and the formulae (1.10) and (1.11) yields the following bilinear generating function

$$
\begin{aligned}
& \mathrm{e}^{(y+w) t} x^{A} z^{B}{ }_{o} F_{k}\left[-; \frac{A+I}{k}, \frac{A+2 I}{k}, \cdots, \frac{A+(k-1) I}{k} ;-(k x)^{k}\right] \\
& \times_{o} F_{k}\left[-; \frac{B+I}{k}, \frac{B+2 I}{k}, \cdots, \frac{B+(k-1) I}{k} ;-t(l z)^{k}\right] \\
& =\Gamma(A+I) \Gamma(B+I) \sum_{n=0}^{\infty} L_{n}(x, y ; k, A) \times L_{n}(z, w ; l, B) \frac{t^{n}}{n !} .
\end{aligned}
$$

In [14], the following definition of Laguerre matrix polynomials has been in- 
troduced:

$$
L_{n}^{(A, \lambda)}(x)=\sum_{s=0}^{\infty} \frac{(-1)^{s}}{s !(n-s) !}(A+I)_{n}\left[(A+I)_{s}\right]^{-1}(\lambda x)^{s}, \lambda \geq 0,
$$

where $A$ be a matrix in $\mathbb{C}^{N \times N}, \alpha \in \sigma(A)$ and $(-\alpha)$ is not an eigenvalue of $A$ for every integer $\alpha>0$ and $\lambda$ be a complex number whose real part is positive. Such matrix polynomials have the following operational representation [14]:

$$
L_{n}^{(B, \lambda)}(z)=\frac{\lambda^{-B}(B+I)_{n}}{n !}\left(1-z \hat{D}_{\lambda}^{-1}\right)^{n}\left\{\lambda^{B}\right\} .
$$

Let us consider the generating relation

$$
f^{(A, B)}(x, y, z, \lambda \mid t)=\sum_{n=0}^{\infty} L_{n}(x, y ; k, A) \times L_{n}^{(B, \lambda)}(z) t^{n} .
$$

Now, directly from (2.4) and (3.1) by employing the previously outlined method leading to the bilinear generating function, we obtain from (3.2) the following bilateral generating function

$$
\begin{aligned}
& \sum_{m=0}^{\infty} \frac{(B+I)_{m}}{m !} y^{m} x^{A} \lambda^{B}{ }_{1} F_{k}\left[-m ; \frac{A+I}{k}, \frac{A+2 I}{k}, \cdots, \frac{A+(k-1) I}{k} ; \frac{(k x)^{k}}{y}\right] \\
& \times{ }_{2} F_{0}\left[-B, m I ;-; \frac{-z}{\lambda}\right]=\sum_{n=0}^{\infty} L_{n}(x, y ; k, A) \times L_{n}^{(B, \lambda)}(z) t^{n} .
\end{aligned}
$$

Similarly, from the operational representation of the two variable Hermite matrix polynomials $H_{n}(x, y, A)$ (see [10])

$$
H_{n}(x, y, A)=\exp \left(-y(2 A)^{-1} \frac{\partial^{2}}{\partial x^{2}}\right)(x \sqrt{(2 A)})^{n},
$$

and (2.4), we can easily derive the following bilateral generating function

$$
\begin{aligned}
& \sum_{s=0}^{\infty} \frac{(-z y t \sqrt{(2 B)})^{s}}{s !}{ }_{1} F_{k}\left[-s ; \frac{A+I}{k}, \cdots, \frac{A+(k-1) I}{k} ; \frac{(k x)^{k}}{y}\right] \\
& \times_{2} F_{0}\left[\frac{-s}{2}, \frac{-s+1}{2} ;--; \frac{-4 w(2 B)^{-1}}{z^{2}}\right] \\
& =\Gamma(A+I) \sum_{n=0}^{\infty} L_{n}(x, y ; k, A) \times H_{n}(z, w, B) \frac{t^{n}}{n !} .
\end{aligned}
$$

Theorem 3.1. Let $A$ and $B$ be a matrices in $\mathbb{C}^{N \times N}$ satisfying the conditions $\mathfrak{R}(\mu)>-1$ for every $\mu \in \sigma(A)$ or $\mu \in \sigma(B), A B=B A$, and $k \in \mathbb{Z}^{+}$. Then

$$
\begin{aligned}
& L_{n}(x, y ; k, A) \times L_{m}(x, y ; k, A) \\
& =\left[\Gamma^{-1}(A+I)\right]^{2} x^{2 A} y^{m+n}{ }_{1} F_{k}\left[-m-n ; \frac{A+I}{k}, \cdots, \frac{A+k I}{k} ; \frac{x^{k}}{k^{k} y}\right],
\end{aligned}
$$

where ${ }_{1} F_{k}$ is defined by (1.9). 


$$
\begin{aligned}
& L_{n}(x, y ; k, A) \times L_{n}(z, w ; l, B) \\
& =\Gamma^{-1}(A+I) \Gamma^{-1}(B+I) \sum_{s=0}^{\infty} \frac{(-1)^{s}(-n I)_{s}(y w)^{n-s} x^{A+k s I} z^{B+l s I}}{s ! k^{k s} l^{l s}} \\
& \times\left[\prod_{j=1}^{k}\left(\frac{A+j I}{k}\right) \prod_{s=1}^{l}\left(\frac{B+j I}{l}\right)_{s}\right]^{-1} \\
& \times F_{0: k ; l}^{1: 0 ; 0}\left[-n+s:---------;---------; x^{k}\right. \\
& ---: \Delta(k ; A+I)+s I ; \Delta(l ; B+I)+s I ; \frac{z^{l}}{y k^{k}},
\end{aligned}
$$

where $F_{0: k ; l}^{1: 0 ; 0}$ is defined by (1.8).

Proof. According to the operational representation (2.4), we have

$$
L_{n}(x, y ; k, A) \times L_{n}(z, w ; l, B)=\left[\Gamma^{-1}(A+I)\right]^{2}\left(y-\hat{D}_{x}^{-k}\right)^{m+n}\left\{x^{2 A}\right\}
$$

which in view of (1.2), the operator in (1.11) and the definition of Pochhammer symbol (1.2), yields the right-hand side of Equation (3.4). Similarly, one can prove the result (3.3)

\section{References}

[1] Bin-Saad, M.G. and Al-Sayaad, A.A. (2015) Study of Two Variable Matrix Laguerre via Symbolic Operational Images. Asian Journal of Mathematics and Computer Research, 2, 42-50.

[2] Durán, A.J. and Van Assche, W. (1995) Orthogonal Matrix Polynomials and Higher Order Recurrence Relations. Linear Algebra and Its Applications, 219, 261-280.

https://doi.org/10.1016/0024-3795(93)00218-O

[3] Geronimo, J.S. (1981) Matrix Orthogonal Polynomials in the Unit Circle. Journal of Mathematical Physics, 22, 1359-1365. https://doi.org/10.1063/1.525073

[4] Jódar, L., Company, R. and Navarro, E. (1994) Laguerre Matrix Polynomials and System of Second Order Differential Equations. Applied Numerical Mathematics, 15, 53-63. https://doi.org/10.1016/0168-9274(94)00012-3

[5] Jódar, L. and Company, R. (1996) Hermite Matrix Polynomials and Second Order Matrix Differential Equations. Approximation Theory and Its Applications, 12, 20-30.

[6] Sinap, A. and Van Assche, W. (1996) Orthogonal Matrix Polynomials and Applications. Journal of Computational and Applied Mathematics, 66, 27-52. https://doi.org/10.1016/0377-0427(95)00193-X

[7] Steffensen, J.F. (1941) The Poweriod, an Extension of the Mathematical Notion of Power. Acta Mathematica, 73, 333-366. https://doi.org/10.1007/BF02392231

[8] Defez, E. and Jódar, L. (1998) Some Applications of the Hermite Matrix Polynomials Series Expansions. Journal of Computational Applied Mathematics, 99, 105-117. https://doi.org/10.1016/S0377-0427(98)00149-6

[9] Durán, A.J. (1996) Markovs Theorem for Orthogonal Matrix Polynomials. Canadian Journal of Mathematics, 48, 1095-1180. https://doi.org/10.4153/CJM-1996-062-4

[10] Batahan, R.S. (2006) A New Extension of Hermite Matrix Polynomial and Its Applications. Linear Algebra and Its Applications, 419, 82-92. https://doi.org/10.1016/j.laa.2006.04.006

[11] Dattoli, G. (2001) Pseudo Laguerre and Pseudo Hermite Polynomials. Atti. Accad. 
Naz. Lincei Cl. Sci. Fis. Mat. Natur. Rend. Lincei (9) Mat. Appl., 12, 75-84.

[12] Defez, E. and Jódar, L. (2002) Chebyshev Matrix Polynomials and Second Order Matrix Differential Equations. Utilitas Mathematica, 62, 107-123.

[13] Jódar, L., Company, R. and Ponsoda, E. (1995) Orthogonal Matrix Polynomials and Systems of Second Order Differential Equations. Differential Equations and Dynamical Systems, 3, 269-288.

[14] Jódar, L. and Sastre, J. (1998) On the Laguerre Matrix Polynomials. Utilitas Mathematica, 53, 37-48.

[15] Jódar, L. and Defez, E. (1998) On Hermite Matrix Polynomials and Hermite Matrix Function, Approximation. Theory and Its Applications, 14, 36-48.

[16] Metwally, M.S., Mohamed, M.T. and Shehata, A. (2010) On Pseudo Hermite Matrix Polynomials of Two Variables. Banach Journal of Mathematical Analysis, 4, 169-178. https://doi.org/10.15352/bjma/1297117251

[17] Miller, K.S. and Ross, B. (1993) An Introduction to Fractional Calculus and Fractional Differential Equations. J. Wiley and Sons, New York.

[18] Pathan, M.A., Bin-Saad, M.G. and Al-Sarhi, F. (2015) On Matrix Polynomials Associated with Hermite Matrix Polynomials. Tamkang Journal of Mathematics, 46, 167-177. https://doi.org/10.5556/j.tkjm.46.2015.1722

[19] Sayyed, K.A.M., Metwally, M.S. and Batahan, R.S. (2004) Gegenbauer Matrix Polynomials and Second Order Matrix Differential Equations. Divulgaciones Matematicas, 12, 101-115.

[20] Defez, E. and Jódar, L. (2004) Jacobi Matrix Differential Equation, Polynomial Solutions, and Their Properties. Computers and Mathematics with Applications, 48, 789-803. https://doi.org/10.1016/j.camwa.2004.01.011

[21] Varma, S., Cekim, B. and Tasdelen Yesildal, F. (2011) On Konhauser Matrix Polynomials. Ars Combinatoria, 100, 193-204.

[22] Jódar, L. and Defez, E. (1996) Some New Matrix Formulas Related to Hermite Matrix Polynomials Theory. Proceedings of the International Workshop on Orthogonal Polynomials in Mathematical Physics, Madrid, 24-26 June 1996.

[23] Dattoli, G. (2000) Hermite-Bessel and Laguerre-Bessel Functions: A Byproduct of the Monomiality Principle. Advanced Special Functions and Applications, 1, 147-164.

[24] Srivastava, H.M. and Karlsson, W. (1985) Multiple Gaussian Hypergeometric Series. Ellis Horwood Limited, Chichester, New York.

[25] Dattoli, G., Mancho, A.M., Quattromini, A. and Torre, A. (2001) Generalized Polynomials, Operational Identities and Their Applications. Radiation Physics and Chemistry, 57, 99-109. https://doi.org/10.1016/S0969-806X(00)00426-6 